\title{
Valor da liquidez: análise em empresas brasileiras ISE e não ISE
}

\section{Raquel Alves dos Santos Calda ${ }^{1}$, Ercílio Zanolla ${ }^{2}$, Lúcio de Souza Machado ${ }^{2}$ e Luiz Carlos da Silva Oliveira ${ }^{2}$}

\author{
${ }^{1}$ Universidade Federal de Goiás. Faculdade de Administração, Ciências Contábeis e \\ Econômicas. Graduação em Ciências Contábeis. Campus II. Caixa Postal 131. \\ Goiânia-GO, Brasil (CEP 74001-970). E-mail: raquel.alvesdsc@gmail.com. \\ ${ }^{2}$ Universidade Federal de Goiás. Faculdade de Administração, Ciências Contábeis e \\ Econômicas. Campus II. Caixa Postal 131. Goiânia-GO, Brasil (CEP 74001-970).
}

Resumo. Este estudo buscou identificar a relação entre a classificação de sustentabilidade das empresas brasileiras em ISE (índice de sustentabilidade empresarial) e não ISE, e o desempenho financeiro mensurado pelo valor da liquidez (vliq). Analisou-se 28 empresas, 11 ISE e 18 não ISE, no período de 2012 a 2016. A análise dos resultados pautou-se na estatística descritiva e no Teste de Mann-Whitney para duas amostras independentes. As empresas do grupo ISE apresentaram maior vliq estatisticamente significante a $5 \%$. Conclui-se que as empresas com gestão sustentável podem ter maior desempenho financeiro através da gestão eficiente do capital de giro mensurado pelo vliq.

Palavras-chave: Valor da liquidez; Sustentabilidade; ISE; Desempenho.

\begin{abstract}
Liquidity value: Analysis in the Brazilian's companies ISE and non-ISE. This study sought to identify the relationship between the sustainability classification of Brazilian companies in ISE (corporate sustainability index) and non-ISE, and the financial performance measured by the value of liquidity (vliq). Twenty-eight companies were analyzed, eleven ISE and eighteen non-ISE, in the period from 2012 to 2016. The analysis of the results was based on descriptive statistics and the MannWhitney Test for two independent samples. The companies in the ISE group had a higher statistically significant vliq at 5\%. It is concluded that companies with sustainable management can have greater financial performance through the efficient management of working capital measured by the vliq.
\end{abstract}

Keywords: Liquidity value; Sustainability; ISE; Performance.
Recebido

$31 / 05 / 2021$

Aceito

$08 / 08 / 2021$

Disponível on line

$11 / 08 / 2021$

Publicado

$31 / 08 / 2021$

Acesso aberto

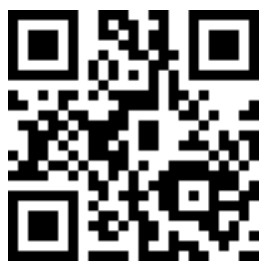

\footnotetext{
(1) 0000-0002-0902-4725

Raquel Alves dos Santos Calda

(D) $0000-0002-7371-427 \mathrm{X}$ Ercílio Zanolla

(1) 0000-0003-4434-2830

Lúcio de Souza

Machado
} 


\section{Introdução}

O capital de giro garante a sustentabilidade da atividade operacional (Assaf Neto e Silva, 2012) e para Uremadu et al. (2012) "nos últimos anos, a gestão do capital de giro ganhou importância à medida que gestores e acadêmicos reconhecem a importância da eficiente gestão da liquidez de uma empresa como vital para a sobrevivência da empresa, especialmente em um momento de turbulência financeira global". No entanto, para Wang et al. (2020, p. 2) "a importância da sustentabilidade financeira corporativa reside no fato de que também afeta diretamente os demais dois pilares (social e ambiental) da sustentabilidade". Para Sachs (2004) a evolução econômica tem influências positivas nos aspectos sociais e ambientais.

Nesse sentido, o relatório "Nosso Futuro Comum", elaborado pela Comissão Mundial para o Meio Ambiente e o Desenvolvimento (CMMAD), apresenta informações sobre questões sociais e ambientais e define desenvolvimento sustentável como "aquele que atende as necessidades do presente sem comprometer as possibilidades de gerações futuras atenderem suas próprias necessidades" (CMMAD, 1991, p. 9).

Para o Ethos Instituto de Pesquisa Aplicada e Instituto Ethos de Responsabilidade Social (ETHOS, 2009), "a sustentabilidade empresarial consiste em assegurar o sucesso do negócio e ao mesmo tempo contribuir para o desenvolvimento econômico e social da comunidade, um meio ambiente saudável e uma sociedade estável". Assim, infere-se que implementar uma gestão sustentável pode se tornar uma oportunidade para que as empresas consigam obter melhores resultados. Por exemplo, a maximização do uso de recursos naturais por meio de práticas de aproveitamento de materiais, como a reutilização ou reciclagem de produtos ea redução de desperdícios (água e energia), podem influenciar em aumento na lucratividade (Pimenta, 2010).

Com base nesses argumentos do ETHOS (2009) e de Pimenta (2010), pode-se compreender que a sustentabilidade está relacionada ao progresso da empresa, sem prejudicar o meio ambiente e a sociedade. Desse modo, os investidores começaram a identificar o quadro ambiental como um novo tipo de risco aos seus retornos financeiros que, consequentemente, poderia afetar o desempenho da empresa.

Por essa razão, despertou-se no mercado um movimento aos Investimentos Sustentáveis Responsáveis. As empresas com enfoque sustentável são mais hábeis para enfrentar os riscos econômicos e gerar um retorno de longo prazo (Moraes et al., 2014).

Nessa concepção, nos Estados Unidos, em 1999, foi criado o índice Dow Jones Sustainability Index (DJSI) composto por empresas definida como sustentáveis. Em seguida, começaram a surgir outros índices como da Bolsa de Londres, em 2001 FTSE4Good. Em 2004, foi criado, pela bolsa de Johanesburgo, África do Sul, o índice de JSE (Marcondes e Bacarji, 2010).

No Brasil, o Índice de Sustentabilidade Empresarial (ISE) foi criado em 2005 pela Bolsa de Valores, Mercadorias \& Futuros de São Paulo (atualmente B3). A carteira ISE é composta somente pelas principais empresas nacionais que seguem o empreendimento sustentável e tem renovação anual. O objetivo do ISE é o de incentivar a qualidade, transparência e prestação de contas, responsabilidade com o desenvolvimento sustentável e, como consequência, o desempenho empresarial (B3, 2017). 
Entretanto, questiona-se se as empresas do grupo ISE, efetivamente, apresentam um melhor desempenho financeiro com relação às que não aplicam tais práticas sustentáveis. Porém, a prevenção de degradação do meio ambiente e aos sistemas sociais incorrem em custos e aumentos de gastos. Contudo, essas práticas podem resultar em benefícios, como riscos verificados, redução de acidentes em obrigações ambientais e melhoria no procedimento de produção (Costa, 2007). Para tanto, a liquidez é uma forma de verificar o desempenho e continuidade da empresa (Zanolla e Silva, 2017b), pois empresas com responsabilidade ambiental e social requerem uma gestão mais eficiente.

Também, nesse sentido, Fonseca e Ceretta (2012) afirmam que a sustentabilidade da empresa requer liquidez equilibrada de forma que não prejudique a rentabilidade, ou seja, é preciso aumentar o lucro e maximizar ovalor da empresa. Para Pimentel et al. (2005), a continuidade dos empreendimentos depende que a manutenção da liquidez esteja em equilíbrio e constante.

\section{Sustentabilidade empresarial}

Para Gomes e Tortato (2011) sustentabilidade empresarial pode ser compreendida como desenvolvimento empresarial sustentável, assegurando necessidades humanas presentes sem desprover as gerações futuras. Assim, pode-se inferir que a sustentabilidade busca a continuidade qualitativa dos empreendimentos concomitante com a sociedade.

Já para Marcondes e Bacarji (2010), a missão da empresa não é exclusivamente produzir lucro. A missão deve ir muito além, ou seja, a empresa deve produzir cada vez mais lucro e bem-estar para sociedade, garantido uma longevidade para os negócios e para a vida.

Bassetto (2007) enfatiza a sustentabilidade como um elemento determinador para busca do crescimento econômico, tornando-se relevante no mercado competitivo, particularmente em relação ao desenvolvimento da sociedade e utilização dos recursos naturais conscientizados. Neste sentido, as empresas precisam buscar um equilíbrio entre o desenvolvimento econômico e a utilização de recursos naturais com responsabilidade.

O ETHOS (2012) define responsabilidade social empresária como uma gestão ética e transparente com fixação de metas que incentivam o desenvolvimento sustentável, sobretudo, preservando os recursos ambientais e culturaispropiciando a diminuição das desigualdades sociais.

\section{Índice de Sustentabilidade Empresarial - ISE}

A criação do ISE pela B3, em 2005, foi motivada pela busca de um índice que servisse de modelo para os Investimentos Sociais Responsáveis (ISR) e para a construção de uma carteira de empresas em desenvolvimento sustentável. O ISE foi pioneiro na América Latina subsidiado pela InternationalFinance Corporation (IFC), membro do Grupo Banco Mundial. Atualmente, o Conselho Deliberativo do ISE (CISE) é formado por onze instituições, B3, ABRAPP, ANBIMA, APIMEC, GIFE, IBRACON, IBGC, IFC, Instituto ETHOS, Ministério do Meio Ambiente e PNUMA.

O ISE é uma forma de proporcionar incentivos para as empresas integrarem nas decisões de investimentos questões ambientais, sociais e de governança, ao colaborar para a qualidade de uma cultura sustentável. É composto por empresas representantes de um grupo corporativo sustentável listadas na B3 e tem sua carteira ISE revalidada a cada ano (Marcondes e Bacarji, 2010).

De acordo com a B3 (2017), o Conselho Deliberativo do ISE seleciona até 40 empresas para integrarem a carteira do ISE, com os seguintes critérios de inclusão: ocupar uma das 200 ações como maior Índice de Negociabilidade (IN) no período das três carteiras anteriores, ter negociação de pregão de 50\% ocorridos nas três últimas carteiras, não ser identificado como Penny Stock, ou seja, cujos ativos possuam cotação inferior a 
$\mathrm{R} \$ 1,00$ (um real), seguir os padrões de sustentabilidade e ter sido selecionada pelo Conselho Deliberativo do ISE.

Por outro lado, os critérios estabelecidos para exclusões da carteira são: deixar de seguir os critérios de inclusão citados; passar por recuperação judicial ou extrajudicial, regime especial de administração temporária ou qualquer outra hipótese definida pela Bolsa e se forem identificados pelo Conselho Deliberativo do ISE (CISE) acontecimentos que tenham alterado de forma significativa o desempenho sustentável das empresas (B3, 2017).

Nesse sentido, empresas pertencentes ao ISE são menos endividadas e expostas ao risco (Teixeira et al., 2011), bem como se apresentam mais sólidas aos efeitos da crise de 2008 (Aquegawa e Souza, 2010).

De outro lado, segundo os autores Macedo et al. (2007), a adesão ao ISE não impacta no desempenho financeiro das empresas. Nunes et al. (2010) verificam que o tamanho e o setor são determinantes a adesão das empresas ao ISE.

Diante o exposto, e também considerando a recessão econômica a partir de 2014, pode-se inferir que as empresas que compõem a carteira ISE tenham se reestruturado no sentido de adequação ao novo cenário com possíveis impactos de gestão, econômicos e de desempenho.

\section{Liquidez: teoria e valor}

Para Sá (2006, p. 237) a eficiência do patrimônio em gerar riquezas para alcançar a capacidade de saldar as obrigações das empresas denomina-se liquidez. 0 mesmo autor compreende o termo liquidez como "a função que os meios patrimoniais exercem para suprir as necessidades de pagamentos".

Para Martins et al. (2012), a análise de liquidez implica em identificar se a empresa apresenta capacidade de saldar compromissos contratuais com os recursos financeiros, materiais, serviços e recursos operacionais.Esses autores destacam, ainda, a importância da liquidez ao afirmar que "tanto a incapacidade de remunerar o capital próprio quanto a de responder pelas suas obrigações significam vida não longa para qualquer sociedade de fins lucrativos" (Martins et al., 2012, p. 123).

Nesse sentido, o gestor financeiro precisa buscar um equilíbrio entre recursos e aplicações de curto e longo prazos. A administração do capital de giro pode ser um procedimento eficiente para melhorar a rentabilidade da empresa, de forma que isso não reflita em uma perda na sua capacidade de pagamento (Fonseca e Ceretta, 2012).

Para Assaf Neto e Silva (2012), a administração de capital de giro influência nas decisões relacionadas às finanças, com informações sobre sua composição, aplicação e gestão dos recursos financeiros e tem como objetivo a continuidade e crescimento da empresa.

O termo administração de capital de giro refere-se ao controle das contas ativo e passivo (circulante) e sua relação. 0 grau de adequação e equilíbrio dessas contas consiste na manutenção dos níveis de rentabilidade e liquidez (Assaf Neto e Silva, 2012). Nesse sentido, corroboram Fonseca e Ceretta (2011) ao estabelecer a manutenção do capital de giro como estratégia fundamental para as atividades empresariais.

Zanolla e Silva (2017a) definem que o capital de giro, como recursos econômicos, pode ser transformado em caixa no curto prazo. Ao deduzir do ativo circulante (AC) o passivo circulante (PC), encontra-se o capital de giro líquido das necessidades de pagamentos a curto prazo. Ainda, aludem que o estudo sobre os elementos do capital de giro é relevante para um melhor entendimento sobre o trade off entre liquidez e rentabilidade.

Para Assaf Neto e Silva (2012, p. 15), o capital de giro líquido ou circulante (CCL) retrata a folga financeira, ao demonstrar os recursos de longo prazo que estão financiando 
os ativos de curto prazo da empresa, ou seja, o capital de giro está relacionado às decisões financeiras de curto prazo na busca do equilíbrio financeiro que

é verificado quando suas obrigações financeiras se encontram lastreadas em ativos com prazos de conversão em caixa similares aos dos passivos. Em outras palavras, o equilíbrio financeiro exige vinculação entre a liquidez dos ativos e os desembolsos demandados pelos passivos Assaf Neto e Silva (2012, p. 15).

Uma questão relevante a ser observada é a distribuição do fluxo financeiro futuro da empresa. Assim, é fundamental observar que os fluxos de recebimento e as necessidades de recursos sejam harmonizáveis pois pode gerar insuficiência de caixa e resultar em situações desfavoráveis como a descontinuidade de operações. Para tanto, é necessário que o ciclo operacional da empresa seja avaliado de forma rigorosa e contínua de modo a garantir desempenho e fluxo de caixa (Assaf Neto e Silva, 2012).

Para Martins et al. (2012), os indicadores de liquidez tradicionais como liquidez seca, corrente, geral e imediata demonstram a situação financeira da empresa, no entanto, estática, e por isso são criticados.

Nesse sentido, pode-se citar, por exemplo, a liquidez corrente que não contempla a essência da teoria da liquidez, ou seja, transformar ativos em caixa. A empresa necessita que seus negócios estejam equiparados entre liquidez e o pagamento de suas obrigações, para atingir e se manter em equilíbrio financeiro (Zanolla e Silva, 2017a).

Considerando o trade off entre liquidez e rentabilidade, Zanolla e Silva (2017b) propõem que a gestão da liquidez pode ser utilizada para criar valor à empresa, conforme modelo descrito no tópico a seguir.

\section{Valor da Liquidez}

Segundo Zanolla e Silva (2017b), a liquidez contábil é definida por intermédio de recursos evidenciados nos balanços, como ativos e passivos circulantes. Para Ross et al. (2009, p. 58) "liquidez refere-se à velocidade e facilidade com a qual um ativo pode ser convertido em caixa". Segundo os mesmos autores "[...] a liquidez possui duas dimensões: facilidade de conversão versus perda de valor". Para tanto, a gestão financeira operacional deve ser considerada, pois o ativo circulante caracteriza-se por curto prazo e conversão rápida dos bens. A dinâmica de giro dos elementos do ativo circulante resulta em menor necessidade de investimento e, consequentemente, muda a forma de financiamento que pode ser através de recursos passivos operacionais, capital próprio ou de terceiros (Assaf Neto, 2007).

Mediante a forma de financiamento de investimento é definido o custo financeiro, que é determinado por $k i$ (capital de terceiros) e $K e$ (capital próprio). Por outro lado, o investimento proporciona à empresa um determinado retorno.

Com isso, e conforme Zanolla e Silva (2017b), é possível quantificar o valor criado pela gestão do capital de giro. 0 modelo proposto pelos autores mensura o valor da liquidez (vliq) a partir do montante de investimento operacional mantido pela empresa e definido por meio do modelo dinâmico do capital de giro e o retorno desse investimento ao considerar o ciclo financeiro e o custo financeiro. Assim, o cálculo do vliq é definido pela diferença entre o retorno da liquidez e o custo da liquidez.

Para definir o custo da liquidez (cliq), é necessário ter a taxa do custo financeiro e o montante de investimento operacional que requer recursos onerosos, necessidade de capital de giro (NCG). A taxa do custo financeiro pode ser estabelecida pelo custo do capital de terceiros (Ki), pelo custo do capital próprio (Ke), CAPM ou também pode ser obtida com dados dos balanços contábeis. 
Como a necessidade de financiar o montante do investimento operacional (NCG) depende da dinâmica do capital de giro, definida pelo ciclo financeiro, primeiro, calcula-se o custo do ciclo financeiro (equação 1)e, em seguida, o custo da liquidez (equação 2):

$$
\begin{array}{ll}
c c f=\frac{T x}{360} \times c f & \text { Equação (1) } \\
c l i q=c c f \times n c g & \text { Equação (2) }
\end{array}
$$

Onde:

$c c f=$ custo do ciclo financeiro

$T x=$ taxa do custo financeiro (anual)

$c f=$ ciclo financeiro (em dias)

$n c g=$ necessidade de capital de giro

O primeiro passo para o cálculo do retorno da liquidez é definir o montante de investimento operacional. Para tanto, Zanolla e Silva (2017b) utilizam o modelo dinâmico de capital de giro e consideram os principais elementos do capital de giro. Assim, têm-se:

Modelo dinâmico do capital de giro:

$$
\mathrm{ncg}=\mathrm{aco}-\mathrm{pco}
$$

Onde:

ncg $=$ necessidade de capital de giro

aco $=$ ativo circulante operacional

pco $=$ passivo circulante operacional

Considerando-se os principais elementos:

$$
n c g=c+e-f \quad \text { Equação (3) }
$$

Onde:

$\mathrm{c}=$ clientes

$\mathrm{e}=$ estoque

$\mathrm{f}=$ fornecedores

então

aco $=$ clientes e estoques

pco $=$ fornecedores

A NCG representa o montante de investimento operacional que requer financiamento com recursos onerosos de curto ou longo prazo. Como o montante de NCG (investimento operacional) é influenciado pela gestão financeira, calcula-se o retorno que esse investimento proporcionaao considerar o ciclo financeiro.

De acordo com Zanolla e Silva (2017b), o ciclo financeiro está relacionado ao tempo do ciclo operacional que é financiado por recursos financeiros onerosos. Assim, o 
ciclo financeiro sintetiza e incorpora o comportamento ou políticas de estocagem, recebimento de clientes e pagamento de fornecedores.

Para Silva (1998, p. 90), o retorno sobre o ciclo financeiro incorpora a lucratividade e a liquidez: "Esse índice mostra quanto uma empresa recebe por cada unidade que foi investida no ciclo financeiro", conforme segue:

$$
r=\frac{m}{c f x(1-m)} \quad \text { Equação (4) }
$$

Onde:

$\mathrm{r}=$ retorno sobre o ciclo financeiro

$\mathrm{m}=$ margem de lucro

$\mathrm{cf}=$ ciclo financeiro

Assim, o retorno da liquidez (rliq) é dado por:

$$
r l i q=r x n c g \quad \text { Equação (5) }
$$

Conhecidos o retorno da liquidez e o custo da liquidez, calcula-se o valor da liquidez pela diferença, como segue:

$$
v l i q=r l i q-c l i q \quad \text { Equação (6) }
$$

Assim, o vliq representa uma forma de avaliar e acompanhar o desempenho dos gestores em criar valor para a empresa, por meio da gestão do trade off da liquidez e rentabilidade.

\section{Estudos correlatos}

Aquegawa e Souza (2010) pesquisaram a sustentabilidade financeira com índices de liquidez e ciclo financeiro no período da crise de 2008 com o objetivo de verificar e identificar se as empresas da carteira ISE sofreram menos impactos. Os resultados apontaram para a existência de influências da crise na maioria das empresas dos setores do ISE, no entanto, verifica-se que na carteira ISE, em sua totalidade, os impactos foram minimizados.

Nunes (2010) verificou se há diferenças significativas nos índices contábeis em empresas com prática sustentáveis e as que não são sustentáveis. A análise foi abordada em empresas nos setores de energia elétrica e bancos no período de 2005 a 2009. Os resultados indicam a não existência de diferenças entre esses grupos.

Macedo et al. (2007) verificam o desempenho de empresas da carteira ISE e empresas não ISE dos setores de papel e celulose e siderurgia e metalurgia, referente ao ano de 2005. O estudo teve como objetivo analisar se as empresas de políticas sustentáveis apresentam um desempenho superior. Os resultados mostram que não existem diferenças significativas nos índices de liquidez, endividamento e lucratividade.

Teixeira et al. (2011) analisaram o comportamento do nível de endividamento e risco do mercado por meio de regressão com dados em painel de dois grupos de empresas (tratamento e controle - 94 ISE e 284 Não ISE) sendo três anos antes e três anos depois da criação do ISE (2005). Os resultados indicam que as empresas ISE apresentaram relação estatisticamente negativa com o endividamento e o risco de mercado. 


\section{Problema, objetivo geral e justificativa}

Diante do exposto, pode-se inferir que a partir de uma gestão da liquidez equilibrada e constante é possível criar valor às empresas. Assim, por meio deste estudo, pretende-se responder à seguinte questão de pesquisa: Qual o comportamento da liquidez das empresas que fazem parte da ISE e não ISE da B3?

Tem-se, como objetivo geral, identificar se há diferença do valor da liquidez (vliq) entre empresas sustentáveis (ISE) em relação às outras empresas não consideradas sustentáveis (não ISE) da B3 no período de 2012 a 2016, que contempla anos anteriores e posteriores à crise financeira ocorrida no país em 2014. Assume-se como hipótese de pesquisa, $\mathrm{H}_{0}$, que asempresas ISE apresentam desempenho de liquidez melhor do que as empresas não ISE.

Como justificativa e relevância da pesquisa, destaca-se que assuntos sobre sustentabilidade empresarial e Responsabilidade Social Corporativa (RSC) têm provocado interesse em pesquisas com a ideia de incorporar o compromisso com essas práticas nos resultados corporativos (Gomes e Tortato, 2011). Nesse sentido, o número de pesquisas no ramo contábil e de administração, relacionado ao tema social e ambiental, tem representado grande influência, aumento devido ao interesse dos pesquisadores, entidades de classe contábil, indústrias, governos e outras corporações (Farias, 2008; Uremadu et al., 2012).

Assim, a sustentabilidade pode ser conduzida como vantagem competitiva materializada em estratégias de gestão e, consequentemente, com influências positivas no desempenho da empresa. Destarte, ao mensurar o valor da liquidez, objeto de estudo em empresas ISE e não ISE, também se capta a eficiência da gestão operacional das empresas.

Ainda, o estudo busca contribuir em difundir as discussões sobre o valor da liquidez, explorada inicialmente em pesquisa realizada nas empresas brasileiras do setor de energia elétrica, para a academia (Zanolla e Silva, 2017b), ao ampliar as discussões de criação de valor econômico e gestão para sustentabilidade social e ambiental.

Ademais, a presente pesquisa se alinha aos temas que vem se desenvolvendo no meio acadêmico, porém, se diferencia e inova em relação aos estudos apresentados por buscar uma análise de desempenho com a proxy vliq.

\section{Metodologia}

A população é composta pelas empresas listadas no ISE e não ISE da B3. A escolha dos setores é estabelecida considerando-se a intensidade das empresas que compõem a carteira ISE no período analisado.

Assim, para definição da amostra das empresas, primeiramente analisou-se os segmentos que apresentaram maior participação na carteira ISE no período de 2012 a 2016. Em seguida, formou-se o grupo de empresas não ISE de forma equilibrada. Alguns setores não participaram da composição da carteira ISE em todos os anos, por isso, foram excluídos da amostra. 0 setor de 'instituições financeiras', devido às particularidades dos métodos de evidenciação de informações e de alavancagem financeira, também foi excluído.

Nesse sentido, a amostra final de empresas, em número de 38 companhias abertas, está concentrada nos setores de energia, transporte e serviços e papel e celulose.

Os dados secundários das empresas para o cálculo do vliq, conforme especificações no item 2.3, foram coletados da base Economatica ${ }^{\circledR}$ dos anos de 2012 a 2016. Esses dados foram tabulados no programa Microsoft Excel, para calcular o valor da liquidez (vliq). Em relação ao custo da dívida, utilizou-se como proxy Ki, pois segundo Zanolla e Silva (2017b), a maior parte dos investimentos em capital de giro e NCG é financiada com recursos onerosos de terceiros. 
Em seguida, as empresas foram separadas em grupo ISE e não ISE para verificar o comportamento da distribuição do vliq no período de 2012 a 2016. Para identificar a diferença do valor criado pela liquidez entre empresas que adotam práticas sustentáveis (Grupo ISE) e não sustentáveis (Grupo não ISE) realizou-se o teste de diferença de média.

Os testes estatísticos foram realizados através do programa SPSS (Statistical Package for the Social Sciences). Para definir o teste de diferença de médias, primeiramente testou-se a normalidade dos dados e apurou-se que as amostras têm distribuição não normal. Em seguida, como a amostra de empresas ISE e não ISE são independentes aplicou-se o teste estatístico de Mann-Whitney, para testar a seguinte hipótese: As empresas do grupo ISE e não ISE apresentam igualdade da média do vliq.

\section{Resultados e discussão}

Na análise dos resultados apresentam-se estatística descritiva e a significância do teste de média de Mann-Whitney para duas amostras independentes. No entanto, primeiramente, analisa-se a composição das empresas em grupo ISE e não ISE, conforme a Tabela 1.

Tabela 1.Composição da amostra 2012 a 2016.

\begin{tabular}{|l|r|r|r|r|r|r|}
\hline \multirow{2}{*}{ Segmento } & \multicolumn{2}{|c|}{ ISE } & \multicolumn{2}{c|}{ Não ISE } & \multicolumn{2}{c|}{ Total } \\
\cline { 2 - 7 } & $\mathbf{N}$ & $\mathbf{\%}$ & $\mathbf{N}$ & $\mathbf{\%}$ & $\mathbf{N}$ & $\mathbf{\%}$ \\
\hline Energia elétrica & 8 & $32 \%$ & 17 & $68 \%$ & 25 & $66 \%$ \\
\hline Transporte e Serviços & 2 & $20 \%$ & 8 & $80 \%$ & 10 & $26 \%$ \\
\hline Papel e Celulose & 1 & $33 \%$ & 2 & $67 \%$ & 3 & $8 \%$ \\
\hline Total & 11 & $29 \%$ & 27 & $71 \%$ & 38 & $100 \%$ \\
\hline
\end{tabular}

De acordo com a Tabela 1, das 38 empresas da amostra, 11 são da carteira ISE (29\%) e 27 não ISE (71\%). 0 segmento de Energia Elétrica é o mais representativo, 66\%, com oito empresas ISE e 17 não ISE. 0 de Transporte e Serviços corresponde a $26 \%$ da amostra, sendo duas empresas ISE e oito não ISE. Já o segmento de Papel e Celulose é menos representativo com apenas $8 \%$ das empresas da amostra, sendo uma ISE e duas não ISE.

\section{Análise da estatística descritiva}

A estatística descritiva do vliq das empresas ISE e não ISE pesquisadas está evidenciada conforme a Tabela 2.

Tabela 2.Estatística descritiva do vliq de 2012 a 2016.

\begin{tabular}{|l|c|c|c|c|c|}
\hline & Média & Mediana & Desvio Padrão & Mínimo & Máximo \\
\hline ISE & $-6.519,11$ & $1.505,77$ & $53.628,45$ & $-261.140,00$ & $70.162,64$ \\
\hline Não ISE & $-13.491,51$ & $-353,16$ & $126.481,44$ & $-1.384 .210,19$ & $311.717,67$ \\
\hline
\end{tabular}

Observa-se que a média do vliq é negativa, ou seja, as empresas, no período analisado de 2012 a 2016, em média, não conseguem gerar valor por intermédio da gestão da liquidez, mas no grupo de empresas ISE é bem menor, em torno de $50 \%$ do valor médio das empresas não ISE. Pode-se destacar também o desvio padrão elevado nos dois grupos, no entanto, bem menor no grupo de empresas ISE. Em relação a mediana, destaca-se o comportamento das empresas do grupo ISE com valor positivo. Com isso, pode-se inferir 
que mais empresas do grupo ISE geram valor através da liquidez, embora com valor máximo menor devido, a priori, pela quantidade e representatividade das empresas.

De forma geral, pode se afirmar que as empresas que compõem a carteira ISE tem melhor gestão de liquidez.

Para melhor visualizar e entender o comportamento do valor da liquidez em empresas ISE e não ISE, a Tabela 3 apresentaa estatística descritiva por setor.

Tabela 3. Estatística descritiva do vliq de 2012 a 2016 por setor.

\begin{tabular}{|c|c|c|c|c|c|c|}
\hline & & Média & Mediana & D. Padrão & Mínimo & Máximo \\
\hline ISE & \multirow{2}{*}{ Energia } & $-2.810,30$ & $1.613,82$ & $56.163,14$ & $-261.140,00$ & $70.162,64$ \\
\hline Não ISE & & $-21.461,52$ & $-425,65$ & $159.133,56$ & $-1.384 .210,19$ & $311.717,67$ \\
\hline ISE & \multirow{2}{*}{ Transp. } & $2.782,47$ & $17.507,60$ & $45.675,18$ & $-101.559,96$ & $53.187,09$ \\
\hline Não ISE & & $-2,21$ & 81,48 & $6.198,20$ & $-17.840,66$ & $23.349,53$ \\
\hline ISE & \multirow{2}{*}{ Papel/Cel } & $-47.375,13$ & $-43.642,49$ & $40.834,81$ & $-100.277,65$ & $11.111,97$ \\
\hline Não ISE & & 296,39 & $-1.791,89$ & $6.848,18$ & $-3.265,31$ & $19.546,45$ \\
\hline
\end{tabular}

Em relação à média, observa-se que apenas as empresas do setor Transporte do grupo ISE e as empresas não ISE do setor de Papel e Celulose apresentaram média do vliq positiva. Destaca-se uma média superior nos setores de energia e transporte para as empresas ISE. Nesses setores, as empresas ISE tem uma mediana positiva e bem superior à das empresas não ISE o que sinaliza uma gestão da liquidez mais eficiente.

\section{Resultados do teste de médias}

Primeiramente, conforme Tabela 4, são apresentados os resultados dos testes de normalidade da distribuição do vliq das empresas ISE e não ISE e, também, por setor de atividade. Para amostras de até 30 observações, aplicou-se o teste o teste Shapiro-Wilk e para amostras maiores o Teste de Kolmogorov-Smirnov.

Tabela 4.Teste de normalidade.

\begin{tabular}{|l|c|c|c|c|c|c|}
\hline \multirow{2}{*}{} & \multicolumn{3}{|c|}{ Kolmogorov-Smirnov } & \multicolumn{3}{c|}{ Shapiro-Wilk } \\
\cline { 2 - 7 } & Estatística & $\mathbf{N}$ & Sig. & Estatística & N & Sig. \\
\hline Empresas ISE & 0,254 & 45 & 0,000 & 0,765 & 45 & 0,000 \\
\hline Empresas não ISE & 0,377 & 135 & 0,000 & 0,209 & 135 & 0,000 \\
\hline Energia ISE & 0,339 & 30 & 0,000 & 0,620 & 30 & 0,000 \\
\hline Energia não ISE & 0,368 & 85 & 0,000 & 0,271 & 85 & 0,000 \\
\hline Transporte ISE & 0,212 & 10 & 0,200 & 0,882 & 10 & 0,136 \\
\hline Transporte não ISE & 0,259 & 40 & 0,000 & 0,767 & 40 & 0,000 \\
\hline Papel e Celulose ISE & 0,209 & 5 & 0,200 & 0,979 & 5 & 0,928 \\
\hline Papel e Celulose não ISE & 0,410 & 10 & 0,000 & 0,512 & 10 & 0,000 \\
\hline
\end{tabular}

De acordo com os resultados dos testes de normalidade do vliq, no geral, todas as distribuições apresentam significância $\mathrm{p}<0,05$, ou seja, possuem distribuição não normal. Assim, aplica-se o teste não paramétrico de Mann-Whitney.

Na Tabela 5, evidenciam-se os resultados das médias das empresas que compõem a carteira ISE e não ISE, bem como a estatística e significância do Teste de Mann-Whitney para amostras independentes. 
Tabela 5. Teste de Mann-Whitney do vliq de 2012 a 2016.

\begin{tabular}{|c|c|c|c|c|c|c|}
\hline Grupo & Segmento & $\mathbf{N}$ & $\begin{array}{l}\text { Mean } \\
\text { rank }\end{array}$ & $\begin{array}{c}\text { Sum of } \\
\text { ranks }\end{array}$ & $\begin{array}{c}\text { Test } \\
\text { statistics }\end{array}$ & $\begin{array}{l}\text { Sig. assist. } \\
\text { ( } 2 \text { caudas) }\end{array}$ \\
\hline ISE & \multirow{2}{*}{ Amostra geral } & 45 & 104,09 & $4.684,00$ & \multirow{2}{*}{$2.426,000$} & \multirow{2}{*}{0,043} \\
\hline Não ISE & & 135 & 85,97 & $11.606,00$ & & \\
\hline ISE & \multirow{2}{*}{ Energia } & 30 & 71,77 & $2.153,00$ & \multirow{2}{*}{862,000} & \multirow{2}{*}{0,009} \\
\hline Não ISE & & 85 & 53,14 & $4.517,00$ & & \\
\hline ISE & \multirow{2}{*}{ Transporte } & 10 & 29,80 & 298,00 & \multirow{2}{*}{157,000} & \multirow{2}{*}{0,297} \\
\hline Não ISE & & 40 & 24,43 & 977,00 & & \\
\hline ISE & \multirow{2}{*}{ Papel/celulose } & 5 & 4,80 & 24,00 & \multirow{2}{*}{9,000} & \multirow{2}{*}{0,050} \\
\hline Não ISE & & 10 & 9,60 & 96,00 & & \\
\hline
\end{tabular}

Conforme os resultados apresentados na Tabela 5, no geral, o Teste de MannWhitney é significante, ou seja, estatisticamente, o vliq das empresas do grupo ISE e não ISE é diferente, exceção feita ao Setor de Transporte, em que o Teste de Mann-Whitney não foi significante ao nível de significância de 5\%. Assim, a hipótese do teste que o vliq das empresas ISE é igual ao das não ISE é rejeitada para os Setores de Energia e Papel e Celulose e para todas as empresas da amostra.

No geral, o grupo ISE apresenta uma média de vliq superior ao do grupo não ISE. Em relação aos setores, apenas as empresas de Papel e Celulose apresentam menor média do vliq do grupo ISE, devido provavelmente, a pequena quantidade de empresas. Assim, a hipótese de pesquisa $\mathrm{H}_{0}$ - As empresas ISE apresentam desempenho melhor do que as empresas não ISE não é rejeitada para todas as empresas da amostra e para as do setor de Energia Elétrica, ou seja, aceita-se parcialmente a hipótese.

Na revisão da literatura, Aquegawa e Souza (2010) informa que as empresas da carteira ISE têm impactos minimizados pela crise financeira de 2008; Nunes (2010) identificou que não há diferença entre os grupos; e Macedo et al. (2007) também corrobora evidenciando resultado de não diferença significativa de liquidez, endividamento e lucrativa entre os grupos ISE e Não ISE. Já Teixeira et al. (2011) indicam que as empresas da carteira ISE apresentam relação negativa com o risco de mercado e o endividamento.

Portanto, em função da descrição dos resultados encontrados pode-se inferir que a gestão do capital de giro das empresas ISE é mais eficiente e, consequentemente, apresentam um vliq maior. Assim, esse resultado confirma os estudos de Aquegawa e Souza (2010) e de Teixeira et al. (2011).

Diante do exposto, pode-se inferir que os resultados do presente estudo destacam que com o passar dos anos as empresas ISE conseguem agregar valor através da boa gestão da liquidez. Isso sinaliza para o crescimento e importância da RSC das empresas. As empresas ISE ao apresentar um vliq maior, a priori, evidenciam que buscam uma maior eficiência da gestão financeira com o uso de recursos (matérias, naturais e humanos) e, consequentemente, com reflexos tanto no desempenho como na qualidade de vida da sociedade.

\section{Considerações finais}

O objetivo geral da pesquisa de verificar se as empresas ISE apresentam desempenho de liquidez melhor do que as empresas não ISE foi atingido. Os resultados da presente pesquisa confirmam a revisão da literatura de que as empresas que fazem a adesão a práticas sustentáveis, ou seja, listadas à carteira ISE, apresentam desempenho mais atrativo. 
Em geral, as evidências estatísticas indicam que o vliq médio é diferente entre empresas ISE e empresas não ISE. Apenas para o setor de transporte não se apuraram diferenças estatísticas entre o vliq de empresas ISE e não ISE, ao nível de significância de $5 \%$.

Todas as empresas dos setores do grupo ISE apresentaram médias do vliq superiores ao grupo não ISE, ou seja, tiveram um retorno da liquidez maior com relação aos custos da liquidez. No entanto, destaca-se que as empresas ISE do Setor de Papel e Celulose apresentaram vliq médio menor que o das empresas não ISE; e no setor de transporte não há evidências estatísticas significantes de diferença do vliq médio entre as empresas ISE e não ISE.

Alguns fatores podem explicar esses resultados como o período de recessão econômica. No entanto, os Setores de Energia Elétrica e Transportes e Serviços por serem de necessidade básica não são tão influenciados, o que não acontece, a priori, com o Setor de Papel e Celulose.

Assim, pode-se concluir que as empresas ISE agregam mais valor, ou seja, demostram ter maior capacidade de eficiência na gestão de capital de giro. Também, é possível depreender que as empresas que fazem a adesão as práticas sustentáveis, além de crescimento econômico e social da comunidade, obtém melhores resultados relacionados ao valor da liquidez.

Esse estudo contribuiu com uma nova abordagem acadêmica, ao comparar o vliq em empresas ISE e não ISE, o que difere de pesquisas com essa abordagem. Ademais, confirma que a utilização de práticas sustentáveis representa uma gestão estratégica com influências positivas no desempenho (Macedo et al., 2007; Aquegawa e Souza, 2010; Nunes et al., 2010).

Como limitação da pesquisa pode-se destacar a volatilidade na composição da carteira ISE, ou seja, no período de 2012 a 2016 muitas empresas ingressaram e também deixaram de compor a carteira, sendo necessário excluí-las, o que consequentemente interferiu na quantidade da amostra. Também, pode-se considerar que as inferências estatísticas não possibilitam a análise de causalidade das variáveis.

Sugere-se para pesquisas futuras, reproduzir este estudo para outros setores e utilizar uma série temporal maior, bem como investigar profundamente o desempenho organizacional das empresas, evidenciando as análises de dados em painel e inclusão de variáveis de controle.

\section{Conflito de interesses}

Os autores declaram não haver conflito de interesses.

\section{Referências}

Aquegawa, H. P.; Souza, E. S. Sustentabilidade financeira a partir dos índices de liquidez e ciclo financeiro: uma análise setorial do portfólio ISE frente à crise de 2008. Anais do X Congresso USP de Controladoria e Contabilidade, São Paulo, 2010.

Assaf Neto, A. Estrutura e análise de balanços: um enfoque econômico-financeiro. 8 ed. São Paulo: Atlas, 2007.

Assaf Neto, A.; Silva, C. A. T. Administração do capital de giro. 4. ed. São Paulo: Atlas, 2012.

B3 - Brasil, Bolsa e Balção. Índice de Sustentabilidade Empresarial - ISE. 2017. Disponível em: <http://www.bmfbovespa.com.br/pt_br/produtos/indices/indices-de-sustentabilida de/indice-de-sustentabilidade-empresarial-ise.htm>. Acesso em: 8 mar. 2017. 
Bassetto, L. I. Sustentabilidade empresarial um estudo baseado no relatório de uma concessionária de energia. Ponta Grosa: Universidade Tecnológica Federal do Paraná, 2007. (Dissertação de mestrado).

CMMAD - Comissão Mundial Sobre o Meio Ambiente e Desenvolvimento. Nosso futuro comum. 2. ed. Rio de Janeiro: FGV, 1991.

Costa, F. J. M. Sustentabilidade e desempenho financeiro: uma análise do mercado brasileiro de ações. Salvador: Universidade Federal da Bahia, 2007. (Dissertação de mestrado).

ETHOS - Instituto de Pesquisa Aplicada e Instituto Ethos de Responsabilidade Social. 2009. Disponível em: <http://www3.ethos.org.br>. Acesso em: 21 jun. 2017.

ETHOS - Instituto de Pesquisa Aplicada e Instituto Ethos de Responsabilidade Social. 2012. Disponível em: <http://www3.ethos.org.br>. Acesso em: 21 jun. 2017.

Farias, K. T. R. A relação entre divulgação ambiental, desempenho ambiental e desempenho econômico nas empresas brasileiras de capital aberto: uma pesquisa utilizando equações simultâneas. Ribeirão Preto: Universidade de São Paulo, 2008. (Dissertação de mestrado).

Fonseca, J. L.; Ceretta, P. S. A gestão da liquidez e o seu reflexo no retorno sobre o capital próprio e no lucro por ação das empresas pertencentes à BMF\&BOVESPA. Revista Alcance - Eletrônica, v. 19, n. 2, p. 202-221, 2012.

Gomes, F. P.; Tortato, U. Adoção de práticas de sustentabilidade como vantagem competitiva: evidências empíricas. Revista Pensamento Contemporâneo em Administração, v. 5, n. 2, p. 33-49, 2011.

Macedo, M. A. S.; Sousa, A. C.; Sousa, A. C. C. Desempenho de empresas socialmente responsáveis: uma análise por índices contábil-financeiros. Produção Online, v. Esp., 2007. https://doi.org/10.14488/1676-1901.v7i4.59

Marcondes, A. W.; Bacarji, C. D. ISE: sustentabilidade no mercado de capitais. São Paulo: Report, 2010.

Martins, E.; Diniz, J. D. A.; Miranda, G. J. Análise avançada das demonstrações contábeis: uma abordagem crítica. São Paulo: Atlas, 2012.

Moraes, L. F. S.; Perera, L. C. J.; Milani Filho, M. A. F.; Kerr, R. B. Desenvolvimento sustentável e insolvência: um estudo de empresas brasileiras. Revista de Gestão Ambiental e Sustentabilidade, v. 3, n. 2, p. 39-57, 2014.

Nunes, J. G.; Teixeira, A. J. C.; Nossa, V.; Galdi, F. C. Análise das variáveis que influenciam a adesão das empresas ao Índice BM\&F Bovespa de sustentabilidade empresarial. Revista de Administração e Contabilidade de Unisinos, v. 7, n. 4, p. 328-340, 2010.

Nunes, T. C. S. Indicadores contábeis como medidas de risco e retorno diferenciados de empresas sustentáveis: um estudo do mercado brasileiro. São Paulo: Universidade de São Paulo, 2010.

Pimenta, H. C. D. (Org.). Sustentabilidade empresarial: práticas em cadeias produtivas. Natal: IFRN Editora, 2010. (Dissertação de mestrado).

Pimentel, R. C.; Braga, R.; Casa Nova, S. P. C. Interação entre rentabilidade e liquidez: um estudo exploratório. Revista de Contabilidade do Mestrado em Ciências Contábeis UERJ, v. 10, n. 2, p. 83-97, 2005. https://doi.org/10.12979/rcmccuerj.v10i2.5599 
Ross, S. A.; Westerfield, R. W.; Jaffe, J.; Lamb, R. Princípios de administração financeira. 2. ed. São Paulo: Atlas, 2009.

Sá, A. L. Teoria da Contabilidade. 4. ed. São Paulo: Atlas, 2006.

Sachs, I. Desenvolvimento: includente, sustentável, sustentado. Rio de Janeiro: Garamond, 2004.

Silva, C. A. T. Contribuição ao estudo do capital de giro a partir do retorno sobre o ciclo financeiro. Contabilidade Gestão e Finanças - UnB Contábil, v. 1, n. 2, p. 86-97, 1998.

Teixeira, E. A.; Nossa, V.; Funchal, B. O Índice Empresarial de Sustentabilidade (ISE) e os impactos no endividamento e na percepção de risco. Revista Contabilidade \& Finanças, v. 22, n. 5, p. 29-44, 2011. https://doi.org/10.1590/S1519-70772011000100003

Uremadu, S. O.; Egbide, B.-C.; Enyi, P. E. Working capital management liquidity and corporate profitability among quoted firms in Nigeria evidence from the productive sector. International Journal of Academic Research in Accounting Finance and Management Sciences, v. 2, n.1, p.80-97, 2012. Disponível em: <https://econpapers.repec.org/ RePEc:hur:ijaraf:v:2:y:2012:i:1:p:80-97>. Acesso em: 23 fev. 2021.

Wang, Z.; Akbar, M.; Akbar, A. The interplay between working capital management and a firm's financial performance across the corporate life cycle. Sustainability, v. 12, n. 4, 1661, 2020. https://doi.org/10.3390/su12041661

Zanolla, E.; Silva, C. A. T. Liquidez: efeito do dinamismo é da sincronia dos elementos do capital de giro no desempenho das empresas brasileiras. Revista Contabilidade Vista \& Revista, v. 28, n. 2, p. 30-52, 2017a.

Zanolla, E.; Silva, C. A. T. O valor da liquidez: um estudo exploratório nas empresas brasileiras no setor de energia elétrica. Revista Eletrônica da Administração, v. 86, n. 1, p. 118-136, 2017b.

Informação da Licença: Este é um artigo Open Access distribuído sob os termos da Licença Creative Commons Attribution, que permite uso irrestrito, distribuição e reprodução em qualquer meio, desde que a obra original seja devidamente citada. 